\title{
A Modified Sequential Monte Carlo Procedure for the Efficient Recursive Estimation of Extreme Quantiles
}

\author{
Serdar Neslihanoglu* \\ Department of Statistics, Faculty of Science and Letters, Eskisehir Osmangazi \\ University, Eskisehir, Turkey \\ Paresh Date \\ Department of Mathematics, College of Engineering, Design and Physical Sciences, \\ Brunel University, London, UK
}

\begin{abstract}
Many applications in science involve finding estimates of unobserved variables from observed data, by combining model predictions with observations. The Sequential Monte Carlo (SMC) is a well-established technique for estimating the distribution of unobserved variables which are conditional on current observations. While the SMC is very successful at estimating the first central moments, estimating the extreme quantiles of a distribution via the current SMC methods is computationally very expensive. The purpose of this paper is to develop a new framework using probability distortion. We use an SMC with distorted weights in order to make computationally efficient inferences about tail probabilities of future interest rates using the Cox-Ingersoll-Ross (CIR) model, as well as with an observed yield curve. We show that the proposed method yields acceptable estimates about tail quantiles at a fraction
\end{abstract}

${ }^{*}$ Corresponding Author [Serdar Neslihanoglu]

Address: Department of Statistics, Faculty of Science and Letters, Eskisehir Osmangazi University, Meselik Yerleskesi, 26480, Eskisehir, Turkey

E-mail: sneslihanoglu@ogu.edu.tr

Phone: $(+90) 2222393750 / 2973$

Fax: $(+90) 2222393578$ 
of the computational cost of the full Monte Carlo.

Keywords: Simulation, Sequential Monte Carlo, Extreme event simulation, Risk analysis

JEL: C32, C44, C53, C61, G12

\section{Introduction}

This study concerns the problem of estimating the latent states of a dynamic system from observed time series data. Such problems arise in many branches of the physical and social sciences, including the geosciences, navigation and econometrics. Filtering is an indefinitely repetitive procedure of merging model predictions and noisy observations for the purpose of making estimates. The estimate of a state at a particular point in time is a probability distribution (conditional upon the observations which have been made up to that time) which is, in some cases, characterized by its first few central moments. The Kalman Filter (Kalman, 1960) is a commonly used estimator for linear systems. Provided that the conditional densities are Gaussian and that the Kalman Filter is used as a closed-form solution, the Kalman Filter is most satisfactory.

When a system is nonlinear, a stochastic partial differential equation solution must typically be solved in order to generate a conditional distribution. A numerical solution for such an equation is often intractable. This is especially the case when a real-time solution is being sought (e.g., in navigation and tracking) or when the state or the observation dimension is high (e.g., in the geosciences or econometrics). Nonlinear filtering in different disciplines requires various Bayesian approximation methods, each of which require concessions to the accurate estimation and computational loads which are particular to those implementations. To illustrate, the Extended Kalman Filter 
(EKF) (Anderson \& Moore, 1979) and the Unscented Kalman Filter (UKF) (Julier \& Uhlmann, 2004), as well as their variants as cited in Date et al., (2008 and 2010) can all be listed as different approximation methods. A sequential Monte Carlo (SMC), also called a particle filter, extends a discrete approximation of conditional density. The SMC can also be used as a recursive procedure for approximation of the conditional density at random together with novel measurement and insight into state dynamics to form discrete approximation of conditional density at the consecutive phase.

Considering the mean approximation and the covariance of the unobservable variables, the SMC is likely to prove more accurate than filtering algorithms which are based upon the linearization of dynamics and/or assumptions about Gaussianity (Gaussian density), such as the UKF. In the SMC, the UKF and the EKF are frequently utilized for the purpose of producing an approximate posterior distribution (e.g., a proposal distribution). The SMC has experienced prominent theoretical advances regarding its asymptotic behaviour when the number of support points (or particles) at each time step tend to infinity (Crisan \& Doucet, 2002; Del Moral et al., 2012) and when the state dimension tends to infinity (Snyder et al., 2008). Significant practical applications of the SMC range from tracking (e.g., Arulampalam et al., 2002), financial mathematics (e.g., Lautier \& Galli, 2004), the geosciences (e.g., Van Leeuwen, 2010) and epidemiology (e.g., Barndorff-Nielsen \& Shephard, 2002), financial econometrics (e.g., Lopes \& Tsay, 2010) and economics (e.g., Sheinson et al., 2014).

On the contrary, the filtering problem has been far less explored in the literature when the propagation of extreme quantiles in the conditional distribution is a greater concern than that of the propagation of its central moments. Arriving at results with extreme probabilities may be fruitful for 
various applications in the context of econometrics (for instance, in determining the probability of the hyperinflation of a financially stable state) and the geosciences (for example, in determining the probability of a rarely occurring extreme weather condition, such as those which typically only occur once every century). A simple simulation method when determining the probability of an order of 1 in 1 million entails producing billions of primary random variable samples. More promising methods can be used for computing tail probabilities which are grounded in importance sampling and the utilization of exponential twisted distributions which entail moving the mean towards the extreme quantile in the event of normal distribution. Kaynar \& Ridder (2010) explore new importance sampling algorithms for the efficient simulation of rare events. Despite this, their methods are not suited to recursive Bayesian computations which are central for the employment of both SMCtype method and time consuming algorithms.

One way of efficiently sampling from the tails of distributions is by distorting the probability distribution by using an appropriate nonlinear weighting function (e.g., Gonzalez \& Wu, 1999; Mattos et al., 2006). Researches investigate the effect of various probability distortion functions for dealing with the estimation of tail quantiles. The use of probability distortion function may be helpful in various applications of many branches of the physical and social sciences, including psychology (for example, in determining the probability distortion by means of a probability weighted function for making decisions under risk (Gonzalez \& Wu, 1999)) and econometrics (for example, in determining the probability distortion for the hedging of futures contracts (Mattos et al., 2006)).

The main objective of this paper is to investigate the use of probability weight distortions for the SMC for the purpose of sampling from tail dis- 
tributions in a recursive fashion. As an application, we demonstrate that the proposed modification can efficiently capture the extreme quantiles of instantaneously compounded interest rate, i.e, the short rate (and hence the extreme quantiles of the corresponding bond yields), when the short rate is driven by the Cox-Ingersoll-Ross (CIR) process (Cox et al., 1985). The proposed procedure can be adapted to other short rate processes, such as those proposed by Vasicek (1977), Chan et al. (1992), and Longstaff \& Schwartz (1993). In fact, there is extensive literature on the use of filtering frameworks in interest rate modelling (e.g., Geyer \& Pichler, 1999; Chatterjee, 2005; Date \& Wang, 2009; De Rossi, 2010; Fileccia, 2012). Our work may be seen as a generic extension of this existing body of work where the SMC is employed to efficiently extract information about very low probability events. Besides modelling interest rates, the proposed modification of the SMC has potential applications in many other branches of science where filtering is employed and where modelling the tail events is of interest. Possible research areas for future exploration include applications in epidemiology and the geosciences.

The rest of the paper is organized as follows. In Section 2, the framework for the sequential Monte Carlo method is outlined along with our novel modification for distorting probability weights. In Section 3, the algorithm is demonstrated with a simulation example. Finally, conclusions are provided in Section 4.

\section{Sequential Monte Carlo (SMC) methods}

Sequential Monte Carlo (SMC) methods for recursive Bayesian estimation are widely discussed in the literature. These methods solve recursive inference 
problem for state space models of the form:

$$
\begin{aligned}
& x_{t}=f_{t}\left(x_{t-1}, v_{t}\right), \\
& y_{t}=h_{t}\left(x_{t}, n_{t}\right) .
\end{aligned}
$$

Here, $x_{t}$ and $y_{t}$ are the state and observation vectors at time $t(t=1, \ldots, T)$, respectively. $v_{t}$ and $n_{t}$ are the state and observation noise vectors at time $t$, respectively. Moreover, $f_{t}$ and $h_{t}$ are possibly non-linear and time-dependent functions of the state and observation vectors at time $t$, respectively.

For the sake of completeness, a variant of this class of methods described in Doucet et al. (2000) is briefly outlined below. The reader is referred to Gordon et al. (1993), Kong et al. (1994), and Doucet \& Johansen (2008) for more details.

\section{Sequential Monte Carlo (SMC)}

At time $t=1,2, \ldots$

1. Importance Sampling

- For $i=1, \ldots, N$

- Sample $\tilde{x}_{t}^{i} \sim \pi\left(x_{t} \mid x_{0: t-1}^{i}, y_{0: t}\right)$ and set $\tilde{x}_{0: t}^{i} \triangleq\left(x_{0: t-1}^{i}, \tilde{x}_{t}^{i}\right)$.

- For $i=1, \ldots, N$

- Evaluate the importance weights up to a normalising constants

$$
\tilde{w}_{t}^{i}=\tilde{w}_{t-1}^{i} \frac{p\left(y_{t} \mid \tilde{x}_{t}^{i}\right) p\left(\tilde{x}_{t}^{i} \mid \tilde{x}_{t-1}^{i}\right)}{\pi\left(\tilde{x}_{t}^{i} \mid \tilde{x}_{0: t-1}^{i}, y_{0: t}\right)} .
$$

- For $i=1, \ldots, N$

- Normalise the importance weights

$$
w_{t}^{i}=\frac{\tilde{w}_{t}^{i}}{\sum_{j=1}^{N} \tilde{w}_{t}^{j}} .
$$


- Evaluate $N_{\text {eff }}$ using

$$
N_{\mathrm{eff}}=\frac{1}{\sum_{i=1}^{N}\left(w_{t}^{i}\right)^{2}} .
$$

2. Re-sampling

- If $N_{\text {eff }} \geqslant N_{\text {threshold }}$

$-x_{0: t}^{i}=\tilde{x}_{0: t}^{i}$ for $i=1, \ldots, N$.

- Otherwise

- For $i=1, \ldots, N$, sample index $j(i)$ distributed according to the discrete distribution with $N$ elements satisfying $\operatorname{Pr}\{j(i)=l\}=\tilde{w}_{t}^{l}$ for $l=1, \ldots, N$.

- For $i=1, \ldots, N, x_{0: t}^{i}=\tilde{x}_{0: t}^{j(i)}$ and $w_{t}^{i}=\frac{1}{N}$.

Given a discrete measure, $\left\{x_{t-1}^{i}, w_{t-1}^{i}\right\}_{i=1}^{N}$ which approximates $p\left(x_{t-1} \mid\right.$ $\left.y_{t-1}\right)$, and a measurement $y_{t}$, this procedure gives the discrete measure $\left\{x_{t}^{i}, w_{t}^{i}\right\}_{i=1}^{N}$ which approximates $p\left(x_{t}, y_{t}\right)$. The convergence results for the SMC can be found in Crisan \& Doucet (2002). The finite sample performance of the SMC depends on the choice of importance density $\pi(\cdot \mid \cdot)$. We will assume in our work that we are utilizing the transition density itself. Moreover, we explicitly introduce a distortion of probabilities in order to emphasize low probability samples. Specifically, we use the following probability distortion function before the re-sampling step in order to generate more particles from the tail quantile.

$$
W_{t}^{i}=\frac{\exp \left(-s \times \tilde{w}_{t}^{i}\right)-1}{\exp (-s)-1} .
$$


Here, $s$ is a user-chosen coefficient and $W_{t}^{i}$ are the modified weights of these particles. Re-sampling with distorted probabilities ensures that extra copies of extreme values of $x_{t}$ are produced at each time step.

As an illustration of the impact of this weighting function, Figure 1 displays the modified weights $W_{t}^{i}$ with various $s$ parameter values $(1,5,10,20$, 50) compared to the standard weights, $\tilde{w}_{t}^{i}$, which are defined in Equation 3. It can clearly be seen that small probabilities will be over-weighted, while high probabilities are still approximately the same when the modified weights

\section{Insert Figure [1]}

$W_{t}^{i}$ are applied compared to the standard weights $\tilde{w}_{t}^{i}$. It is expected that the estimation performance of the extreme values of $x$ at each time with the modified weighted SMC, as defined in the following algorithm, will work better than the standard weighted SMC.

To predict and update Equations 1 and 2, the sequential Monte Carlo (SMC) with modified particle weights using proposed probability distortion (defined in Equation 3) is briefly outlined as follows:

\section{SMC with probability distortion}

At time $t=1,2, \ldots$

1. Importance Sampling

- For $i=1, \ldots, N$

- Sample $\tilde{x}_{t}^{i} \sim \pi\left(x_{t} \mid x_{0: t-1}^{i}, y_{0: t}\right)$ and set $\tilde{x}_{0: t}^{i} \triangleq\left(x_{0: t-1}^{i}, \tilde{x}_{t}^{i}\right)$.

- For $i=1, \ldots, N$

- Evaluate the importance weights up to a normalising constants

$$
\tilde{w}_{t}^{i}=\tilde{w}_{t-1}^{i} \frac{p\left(y_{t} \mid \tilde{x}_{t}^{i}\right) p\left(\tilde{x}_{t}^{i} \mid \tilde{x}_{t-1}^{i}\right)}{\pi\left(\tilde{x}_{t}^{i} \mid \tilde{x}_{0: t-1}^{i}, y_{0: t}\right)} .
$$

- For $i=1, \ldots, N$ 
- Modify weights

$$
W_{t}^{i}=\frac{\exp \left(-s \times \tilde{w}_{t}^{i}\right)-1}{\exp (-s)-1} .
$$

- For $i=1, \ldots, N$

- Normalise modified weights

$$
w_{t}^{i}=\frac{W_{t}^{i}}{\sum_{j=1}^{N} W_{t}^{j}} .
$$

- Evaluate $N_{\text {eff }}$ using

$$
N_{\mathrm{eff}}=\frac{1}{\sum_{i=1}^{N}\left(w_{t}^{i}\right)^{2}} .
$$

2. Re-sampling

- If $N_{\text {eff }} \geqslant N_{\text {threshold }}$

$-x_{0: t}^{i}=\tilde{x}_{0: t}^{i}$ for $i=1, \ldots, N$.

- Otherwise

- For $i=1, \ldots, N$, sample index $j(i)$ distributed according to the discrete distribution with $N$ elements satisfying $\operatorname{Pr}\{j(i)=l\}=w_{t}^{l}$ for $l=1, \ldots, N$.

- For $i=1, \ldots, N, x_{0: t}^{i}=\tilde{x}_{0: t}^{j(i)}$ and $w_{t}^{i}=\frac{1}{N}$.

\section{Simulation Study}

\subsection{Model and methodology}

This section focuses on the simulation study for the purpose of comparing the performance of a sequential Monte Carlo (SMC) with modified (defined 
in Equation 3) and standard weights for estimating the extreme quantiles or expectiles. The Cox-Ingersoll-Ross (CIR) (Cox et al., 1985) model has proved to be very popular for archieving that in both the academic literature and among practitioners due to the three features commonly observed in the data; namely: the nonnegativity of interest rates; conditional heteroscedasticity; and the time-varying market prices of risk (De Rossi, 2010). The CIR model satisfies the following stochastic differential equation:

$$
d x_{t}=\kappa\left(\theta-x_{t}\right) d t+\sigma \sqrt{x_{t}} d Z_{t}
$$

where $x_{t}$ is assumed to be the interest rate and $Z_{t}$ is an independent Wiener process at each time point, $\theta$ is the long-term mean of $x_{t}$, and $\kappa$ is its mean reversion parameter. Moreover, the volatility parameter $\sigma$ determines the magnitude of changes in $x_{t}$.

For this model, the yield of a zero coupon bond which matures after $T$ years is given in the following (give expression for it, with Equation 5, define, $a_{t}, b_{t}$ here). This equation can be used for calibrating the model to the observed yield curve, as well as for finding a yield for a non-standard maturity. More details on the CIR can be found in standard text books, such as Cairns (2004) and Kwok (2008).

There are various methods developed in the financial literature for estimating CIR models. We estimate the following CIR model using the state space approach described by Geyer \& Pichler (1999). This model is considered to be a discretised state space form of the CIR model and is used as an illustrative example (see e.g., Geyer \& Pichler, 1999). The state (transition) equation of this model is given by

$$
x_{t}=\mu_{t}+v_{t} \quad v_{t} \sim N\left(0, Q_{t}\right)
$$

where $\mu_{t}$ and $Q_{t}$ are distributed in such a way that the two moments of the 
approximate normal and exact transition density are equal. The $\mu_{t}$ is defined as

$$
\mu_{t}=\theta[1-\exp (-\kappa \Delta t)]+\exp (-\kappa \Delta t) x_{t-1}
$$

where $\kappa$ and $\theta$ are constants and $\Delta t$ is the time interval between $t$ and $t-1$. The $Q_{t}$ would be defined as

$$
Q_{t}=\sigma^{2} \frac{1-\exp (-\kappa \Delta t)}{\kappa}\left(\frac{\theta}{2}[1-\exp (-\kappa \Delta t)]+\exp (-\kappa \Delta t) x_{t-1}\right)
$$

where $\sigma$ is a constant.

The observation equation represents the linear relationship between the observed yields $\left(y_{t}\right)$ and the state variables $\left(x_{t}\right)$. The observation equation for the observed yields $\left(y_{t}\right)$, characterised by a time to maturity $(\tau)$, is given by

$$
y_{t}=a_{t}+b_{t} x_{t}+n_{t} \quad n_{t} \sim N(0, H)
$$

where $n_{t}$ is a $T \times 1$ vector of measurement errors and $H$ is the variancecovariance matrix of $n_{t}$. The errors $n_{t}$ are serially and cross-sectionally uncorrelated with a zero mean. In our application, the number of observed yields and associated maturities do not change over time. Therefore, $H$ has a constant dimension of $T \times T$ and is assumed to be a diagonal matrix. The $a_{t}$ and $b_{t}$ would be defined as

$$
\begin{aligned}
& a_{t}=-\log \frac{A(\tau)}{\tau}, \\
& b_{t}=\frac{B(\tau)}{\tau}
\end{aligned}
$$

where $\tau$ denotes the maturity associated with the observed yields. The $A(\tau)$ 
and $B(\tau)$ would be defined as

$$
\begin{aligned}
& A(\tau)=\left(\frac{2 \phi_{1} \exp \left(\phi_{2} \frac{\tau}{2}\right)}{\phi_{4}}\right)^{\phi_{3}}, \\
& B(\tau)=\frac{2\left(\exp \left(\phi_{1} \tau\right)-1\right)}{\phi_{4}},
\end{aligned}
$$

where

$$
\begin{aligned}
\phi_{1} & =\sqrt{(\kappa+\lambda)^{2}+2 \sigma^{2}}, \\
\phi_{2} & =\kappa+\lambda+\phi_{1} \\
\phi_{3} & =\frac{2 \kappa \theta}{\sigma} \\
\phi_{4} & =2 \phi_{1}+\phi_{2}\left(\exp \left(\phi_{1} \tau\right)-1\right) .
\end{aligned}
$$

Here, the quantities $A$ and $B$ are the known function of the maturity $(\tau), \lambda$ is the risk premium parameter, and the CIR parameters are presented by $\kappa$, $\theta$ and $\sigma$.

To compare the performance of the SMC with modified (defined in Equation 3) and standard weights, the CIR model simulation for interest rates in the form of Equations 4 and 5 was chosen as an illustrative example, with the following details being obtained from Geyer \& Pichler (1999):

Step 1. Start SMC simulation with the following settings

(a) Parameters

\begin{tabular}{c|c|c|c}
\hline$\kappa$ & $\theta$ & $\sigma$ & $\lambda$ \\
\hline 0.169 & 6.56 & 0.321 & -0.201 \\
\hline
\end{tabular}

(b) Maturity $(\tau)=1$ year, Time Interval $(\Delta t)=\frac{1}{12}$ (Monthly)

(c) Initial value of $x_{0} \sim N(0,1)$. Also, any negative elements of the $x_{t \mid t}$ are replaced with 0 through the simulation period due to the nonnegativity restriction on the state variables: $x_{t}>0$ in the CIR model for interest rates. 
(d) $H$ is chosen as a constant as 1 .

Step 2. Obtain $x_{t}^{i}$ and $y_{t}^{i}$ from step 1 .

Step 3. Repeat step 1 and 2 for each simulation whose details are defined in Table 1.

\section{Insert Table [1]}

Here, the SMC_10K and SMC_100 algorithms use the standard particle weights with 10,000 and 100 particles, respectively while the modified SMC_100 algorithm uses the modified weights of the particles which were discovered by means of the proposed probability distortion (this is defined in Equation 3 with the arbitrarily chosen coefficient $s$ value being equal to 1 , 5, 10, 20, and 50, respectively). Here, all the simulation codes were written using $R$ software version 3.4.2 for Windows 64 bit and was run on a PC with an Intel Core i7 Processor, 16 GB RAM, and a 240 SSD Hard Drive.

The performance of these models are compared with two different measures of the state estimation error: the Mean Absolute Error (MAE) and the Mean Squared Error (MSE). These are defined as follows:

$$
\begin{aligned}
M A E & =\frac{1}{T} \sum_{t=1}^{T}\left|\overline{\widehat{x}}_{t}-\overline{\widehat{x}}_{t, \text { model }}\right| \\
M S E & =\frac{1}{T} \sum_{t=1}^{T}\left(\overline{\widehat{x}}_{t}-\overline{\widehat{x}}_{t, \text { model }}\right)^{2} .
\end{aligned}
$$

Here, $\overline{\widehat{x}}_{t}$ and $\overline{\widehat{x}}_{t, \text { model }}$ are the true average state estimate and model average state estimate obtained from the 500 repeat simulations of each model at each time step $(t=1, \ldots, T)$, respectively. The models with the lowest MAE and MSE values indicate best modelling performance.

\subsection{Results and discussion}

Table 2 displays the MAEs and MSEs for the average state estimate $\left(\overline{\hat{x}}_{t}\right)$ of various quantiles $(5 \%, 10 \%, 90 \%, 95 \%, 100 \%)$ of the CIR as well as the 
normalized computational times for SMC_10K, SMC_100, and modified SMC_100 algorithms with various $s$ parameter values $(1,5,10,20,50)$.

\section{Insert Table [2]}

The results of simulation with seven different models, in terms of the MAE and the MSE for various quantiles, illustrate the following:

1. In terms of accuracy of state estimation, $\mathrm{SMC}_{-} 10 \mathrm{~K}$ is clearly the best algorithm, although it is computationally the most expensive as well.

2. The performance of all the modified SMC_100 methods with different values of $s$ is better than SMC_100, for all the quantiles tested and for both the error measures.

3. The computation times of all the modified SMC_100 methods with different values of $s$ are close to that of SMC_100, with all being around 900 times faster than SMC_10K.

\section{Insert Figures $[2(\mathrm{a})-2(\mathrm{~g})]$}

Figure 2 illustrates the state estimate behaviour at each time step, averaged over 500 simulations, for all the 7 models. It can be seen that increasing the value of $s$ (from SMC_100 to modified SMC_100 $(s=50)$ gradually recovers the accuracy of the state estimate.

Figure 3 displays the density plot of the state estimate $\left(\hat{x}_{t}\right.$, known as an interest rate) particles which were obtained from the results of the 500 repeat simulations with the different limit specifications of the state estimate particles from the SMC_10K (solid line), the SMC_100 (dotted line), and the modified SMC_100 ( $\mathrm{s}=10)$ (dashed line), respectively. Note that the modified SMC_100 with other $s$ parameter values has been omitted for the sake of brevity. Also, the interest rate $\left(\hat{x}_{t}\right)$ is here shown in percentage form.

Insert Figures $[3(\mathrm{a})-3(\mathrm{~d})]$ 
It is worth noting that the sequential Monte Carlo with modified particle weights using the proposed probability distortion (modified SMC_100 $(\mathrm{s}=10))$ provides more robust results than to the sequential Monte Carlo with standard particle weights (SMC_100) when compared to the sequential Monte Carlo using standard weights with 10,000 particles (SMC_10K) for estimating extreme quantile of state estimate $\left(x_{t}\right)$ for the CIR model simulation. Since, it can clearly be seen that the modified SMC_100 (s=10) provides more chance for extreme values to occur in the tail quantiles when compared to the SMC_100.

To summarize, the modified SMC algorithm proposed here offers a means for estimating the tail quantiles of latent states with an accuracy comparable to that of a full Monte Carlo, but at a small fraction of the computational cost. The accuracy of the mean estimates of the modified SMC are still better than what is achievable with the same number of particles using the traditional SMC.

\section{Conclusions}

This paper proposes a modified SMC procedure with a probability distortion at each step. We demonstrate through numerical experiments on a commonly used interest rate model that the modified procedure can estimate tail quantiles accurately and far more efficiently than a full Monte Carlo based SMC. The benefits of the proposed procedure will span a large number of applications across the sciences. It will open up new lines of enquiry and may spur discipline-specific efforts for further research. We are currently exploring the performance improvement of the modified SMC with alternative functions of proposed probability distortions, as well as its application in other domains. For example, the proposed methodology could be 
used to test whether the predicted location of a tracked object, such as a drone, is within a safe flying zone with a given level of confidence.

\section{Acknowledgements}

This work was supported by Eskisehir Osmangazi University Scientific Research Fund (ESOGU BAP), Eskisehir, Turkey [grant number 201519046].

\section{References}

Anderson, B., \& Moore, J. (1979). Optimal Filtering. Prentice-Hall, New Jersey, USA.

Arulampalam, M. S., Maskell, S., Gordon, N., \& Clapp, T. (2002). A tutorial on particle filters for online nonlinear/non-Gaussian Bayesian tracking. IEEE Transactions on Signal Processing, 50(2), 174-188.

Barndorff-Nielsen, O., \& Shephard, N. (2002). Econometric analysis of realized volatility and its use in estimating stochastic volatility models. Journal of the Royal Statistical Society Series B, 64(2), 253-280.

Cairns, A. (2004). Interest Rate Models: An Introduction. Princeton University Press, New Jersey, USA.

Chan, K. C., Karoly, A., Longstaff, F. A., \& Sanders, A. B. (1992). An empirical comparison of alternative models of the short-term interest rate. Journal of Finance, $47(3), 1209-1227$.

Chatterjee, S. (2005). Application of the Kalman filter for estimating continuous time term structure models: The case of UK and Germany. Working papers, Business School - Economics, University of Glasgow. 
Cox, J. C. E., Ingersoll, J., \& Ross, S. (1985). A theory of the term structure of interest rates. Econometrica, 53(2), 385-407.

Crisan, D., \& Doucet, A. (2002). A survey of convergence results on particle filtering methods for practitioners. IEEE Transactions on Signal Processing, 50, 736-746.

Date, P., Jalen, L., \& Mamon, R. (2008). A new algorithm for latent state estimation in nonlinear time series models. Applied Mathematics and Computation, 203, 224-232.

Date, P., Mamon, R., \& Jalen, L. (2010). A partially linearized sigma point filter for latent state estimation in nonlinear time series models. Journal of Computational and Applied Mathematics, 233(10), 2675-2682.

Date, P., \& Wang, C. (2009). Linear Gaussian affine term structure models with unobservable factors: Calibration and yield forecasting. European Journal of Operational Research, 195(1), 156-166.

De Rossi, G. (2010). Maximum likelihood estimation of the Cox-IngersollRoss model using particle filters. Computational Economics, 36(1), 1-16.

Del Moral, P., Doucet, A., \& Jasra, A. (2012). On adaptive resampling strategies for sequential Monte Carlo methods. Bernoulli, 18(1), 252278.

Doucet, A., Godsill, S., \& Andrieu, C. (2000). On sequential Monte Carlo sampling methods for Bayesian filtering. Statistics and Computing, 10(3), 197-208.

Doucet, A., \& Johansen, A. M. (2008). A tutorial on particle filtering and smoothing: Fifteen years later. Technical report, Institute of Statistical 
Mathematics, Japan and Department of Statistics, University of Warwick.

Fileccia, G. (2012). A particle filter approach to parameter estimation in stochastic volatility models with jumps for crude oil market. PhD thesis, Polytechnic University of Milan, Italy.

Geyer, A. L. J., \& Pichler, S. (1999). A state-space approach to estimate and test multifactor Cox-Ingersoll-Ross models of the term structure. Journal of Financial Research, 22(1), 107-130.

Gonzalez, R., \& Wu, G. (1999). On the shape of the probability weighting function. Cognitive Psychology, 38(1), 129-166.

Gordon, N., Salmond, D., \& Smith, A. (1993). Novel approach to nonlinear/non-Gaussian Bayesian state estimation. Radar and Signal Processing, 140(2), 107-113.

Julier, S. J., \& Uhlmann, J. K. (2004). Unscented filtering and nonlinear estimation. Proceedings of the IEEE, 92(3), 401-422.

Kalman, R. (1960). A new approach to linear filtering and prediction problems. Journal of Engineering, 82, 35-45.

Kaynar, B., \& Ridder, A. (2010). The cross-entropy method with patching for rare-event simulation of large markov chains. European Journal of Operational Research, 207(3), 1380-1397.

Kong, A., Liu, J. S., \& H., W. W. (1994). Sequential imputations and Bayesian missing data problems. Journal of the American Statistical Association, 89(425), 278-288. 
Kwok, Y.-K. (2008). Mathematical Models of Financial Derivatives. SpringerVerlag Berlin Heidelberg, 2 edition.

Lautier, D., \& Galli, A. (2004). Simple and extended Kalman filters: An application to term structures of commodity prices. Applied Financial Economics, 14(13), 963-973.

Longstaff, F., \& Schwartz, E. (1993). Implementation of the LongstaffSchwartz interest rate model. Journal of Fixed Income, 3(2), 7-14.

Lopes, H. F., \& Tsay, R. S. (2010). Particle filters and bayesian inference in financial econometrics. Journal of Forecasting, 30(1):168-209.

Mattos, F., Garcia, P., \& Pennings, J. (2006). Probability distortion and loss aversion in futures hedging. Conference on Applied Commodity Price Analysis, Forecasting, and Market Risk Management, April 17-18, 2006, St. Louis, Missouri.

Sheinson, D. M., Niemi, J., \& Meiring, W. (2014). Comparison of the performance of particle filter algorithms applied to tracking of a disease epidemic. Mathematical Biosciences, 255, 21-32.

Snyder, C., Bengtsson, T., Bickel, P., \& Anderson, J. (2008). Obstacles to high-dimensional particle filtering. Monthly Weather Review, 136, 46294640 .

Van Leeuwen, P. (2010). Nonlinear data assimilation in geosciences: an extremely efficient particle filter. Quarterly Journal of the Royal Meteorological Society, 136, 1991-1999.

Vasicek, O. (1977). An equilibrium characterization of the term structure. Journal of Financial Economics, 5(2), 177-188. 


\section{Tables}

Table 1. The simulation details for all algorithms of CIR model

\begin{tabular}{l|c|c|c}
\hline Model & Particles $(N)$ & Time Steps $(T)$ & Repeat $(R)$ \\
\hline SMC_10K & 10,000 & 100 & 500 \\
\hline SMC_100 & 100 & 100 & 500 \\
\hline Modified SMC_100 (s =1) & 100 & 100 & 500 \\
\hline Modified SMC_100 $(\mathrm{s}=5)$ & 100 & 100 & 500 \\
\hline Modified SMC_100 $(\mathrm{s}=10)$ & 100 & 100 & 500 \\
\hline Modified SMC_100 (s=20) & 100 & 100 & 500 \\
\hline Modified SMC_100 $(\mathrm{s}=50)$ & 100 & 100 & 500 \\
\hline
\end{tabular}


Table 2. MAE and MSE of model average state estimate $\left(\overline{\hat{x}}_{t}\right)$ various quantiles of CIR and computational time of model simulations

\begin{tabular}{|c|c|c|c|c|c|c|}
\hline & \multicolumn{5}{|c|}{ MAE } & \multirow{2}{*}{$\begin{array}{c}\text { Computation } \\
\text { Time }\end{array}$} \\
\hline & \multicolumn{5}{|c|}{ Quantile } & \\
\hline Model & $5 \%$ & $10 \%$ & $90 \%$ & $95 \%$ & $100 \%$ & \\
\hline SMC_10K & 0.0021 & 0.0049 & 0.0457 & 0.0491 & 0.0502 & 1 \\
\hline SMC_100 & 0.0023 & 0.0153 & 0.9686 & 1.0945 & 1.2281 & 0.00119 \\
\hline $\begin{array}{l}\text { Modified SMC_100 } \\
(\mathrm{s}=1)\end{array}$ & 0.0021 & 0.0103 & 0.8606 & 0.9800 & 1.1102 & 0.00115 \\
\hline $\begin{array}{l}\text { Modified SMC_100 } \\
(\mathrm{s}=5)\end{array}$ & 0.0019 & 0.0073 & 0.4249 & 0.4860 & 0.5516 & 0.00118 \\
\hline $\begin{array}{l}\text { Modified SMC_100 } \\
(\mathrm{s}=10)\end{array}$ & 0.0017 & 0.0044 & 0.1964 & 0.2170 & 0.2374 & 0.00120 \\
\hline $\begin{array}{l}\text { Modified SMC_100 } \\
(\mathrm{s}=20)\end{array}$ & 0.0020 & 0.0071 & 0.1370 & 0.1538 & 0.1733 & 0.00123 \\
\hline \multirow[t]{3}{*}{$\begin{array}{l}\text { Modified SMC_100 } \\
(\mathrm{s}=50)\end{array}$} & 0.0022 & 0.0053 & 0.2138 & 0.2434 & 0.2715 & 0.00132 \\
\hline & \multicolumn{5}{|c|}{ MSE } & \\
\hline & \multicolumn{5}{|c|}{ Quantile } & \\
\hline Model & $5 \%$ & $10 \%$ & $90 \%$ & $95 \%$ & $100 \%$ & \\
\hline SMC_10K & 0.0002 & 0.0004 & 0.0030 & 0.0032 & 0.0033 & \\
\hline SMC_100 & 0.0002 & 0.0038 & 1.4174 & 1.7398 & 2.1124 & \\
\hline $\begin{array}{l}\text { Modified SMC_100 } \\
(\mathrm{s}=1)\end{array}$ & 0.0002 & 0.0016 & 1.2530 & 1.5393 & 1.8840 & \\
\hline $\begin{array}{l}\text { Modified SMC_100 } \\
(\mathrm{s}=5)\end{array}$ & 0.0002 & 0.0008 & 0.2657 & 0.3426 & 0.4295 & \\
\hline $\begin{array}{l}\text { Modified SMC_100 } \\
(\mathrm{s}=10)\end{array}$ & 0.0002 & 0.0003 & 0.0538 & 0.0624 & 0.0715 & \\
\hline $\begin{array}{l}\text { Modified SMC_100 } \\
(\mathrm{s}=20)\end{array}$ & 0.0001 & 0.0008 & 0.0261 & 0.0318 & 0.0398 & \\
\hline $\begin{array}{l}\text { Modified SMC_100 } \\
(\mathrm{s}=50)\end{array}$ & 0.0002 & 0.0004 & 0.0792 & 0.0971 & 0.1132 & \\
\hline
\end{tabular}

Notes: The computation times of the algorithms are normalized with respect to the computation time of SMC_10K (taken to be 1). 


\section{Figures}

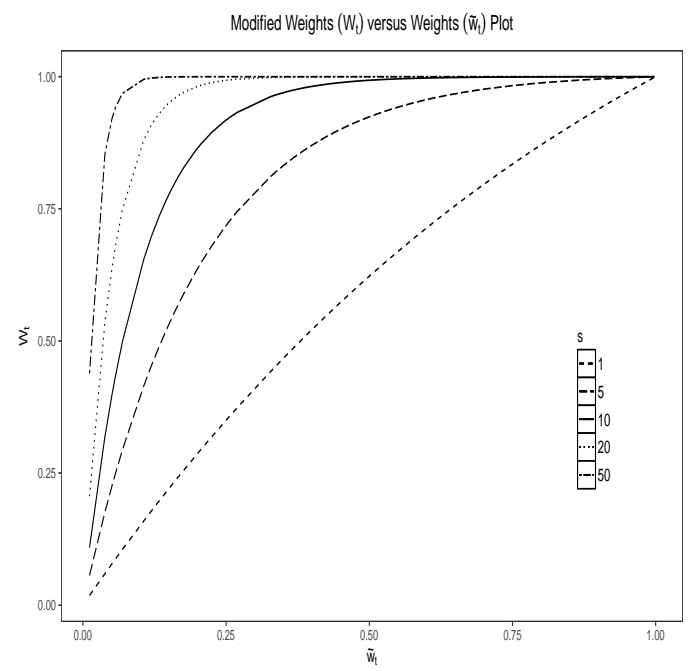

Figure 1. The plot of modified weights with different $s$ parameter values $(1,5,10$, 20, and 50) versus weights defined in Equation 3 are displayed, respectively. 


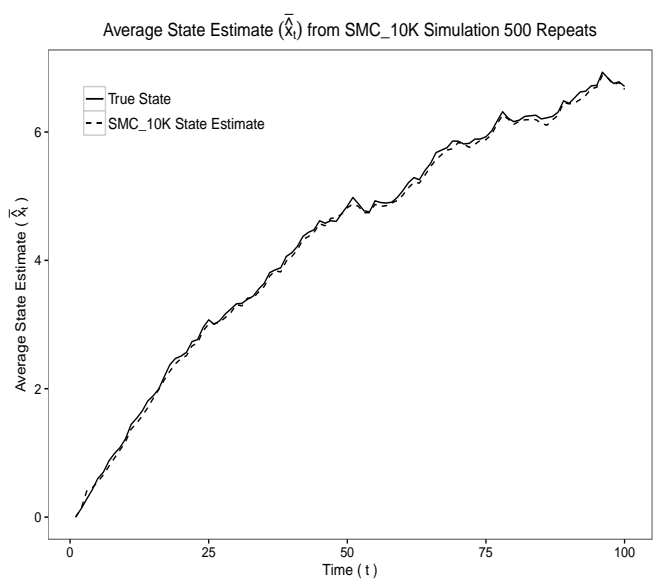

(a) $\mathrm{SMC}_{-} 10 \mathrm{~K}$

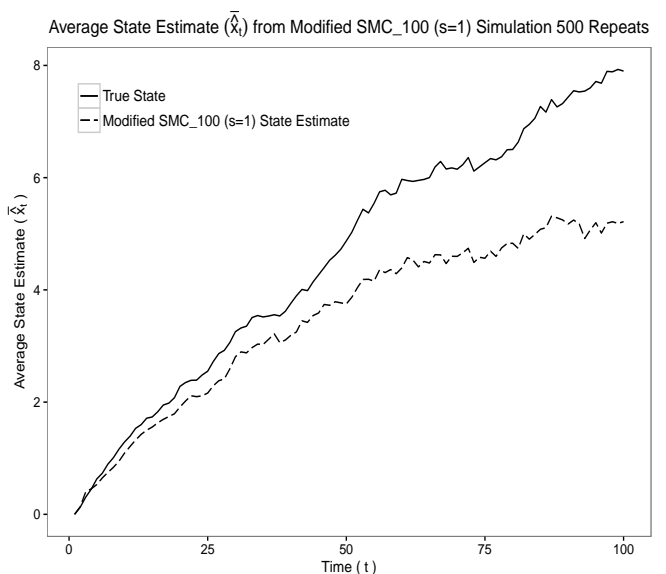

(c) Modified SMC_100 (s=1)

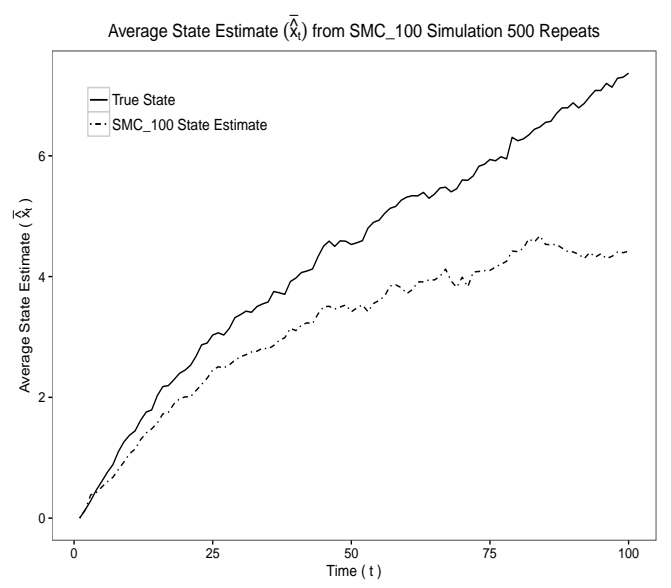

(b) $\mathrm{SMC}_{-} 100$

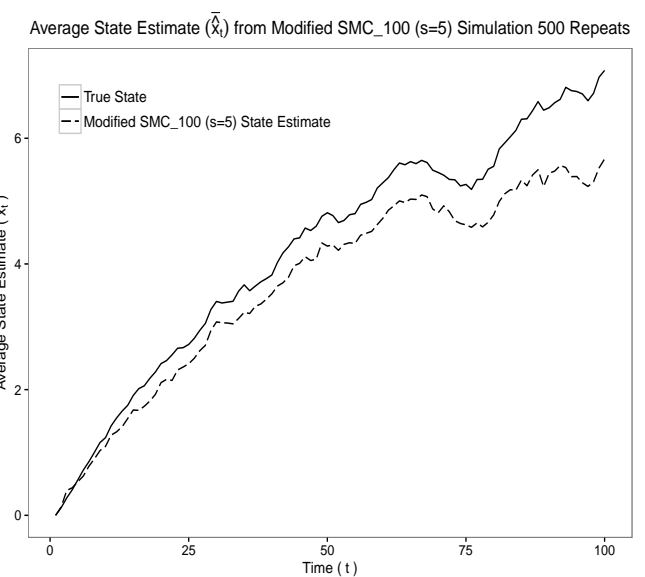

(d) Modified SMC_100 (s=5) 


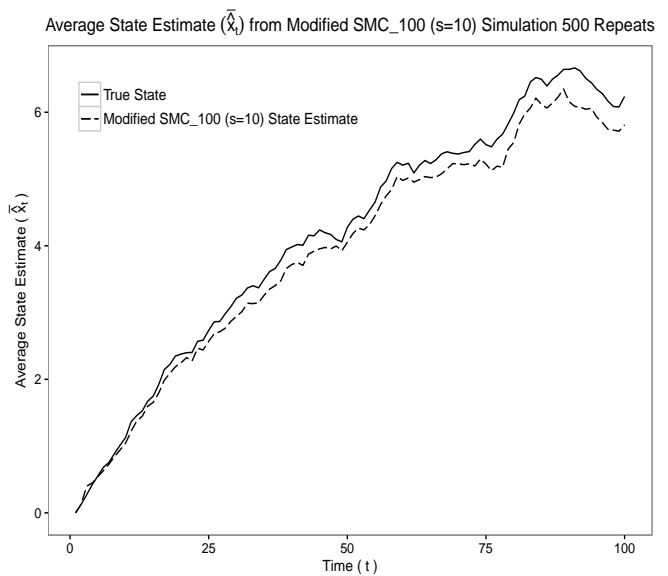

(e) Modified SMC_100 $(\mathrm{s}=10)$

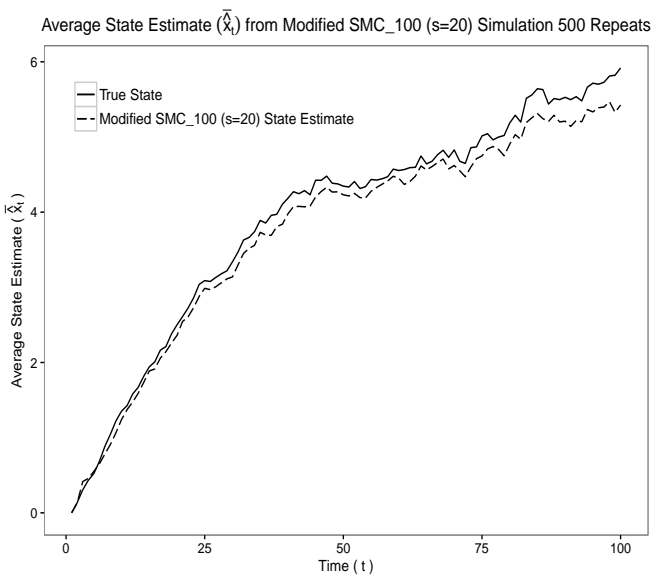

(f) Modified SMC_100 $(\mathrm{s}=20)$

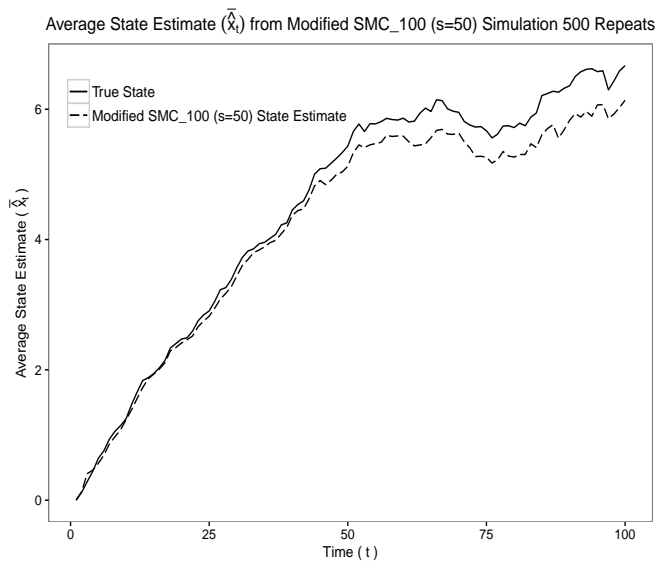

(g) Modified SMC_100 $(\mathrm{s}=50)$

Figure 2. The true state versus the average state estimate $\left(\overline{\hat{x}}_{t}\right)$ for each time step $(t=$ $1, \ldots, T)$ from the SMC_10K (2(a)), the SMC_100 (2(b)), the modified SMC_100 $(\mathrm{s}=1)(2(\mathrm{c}))$, the Modified SMC_100 $(\mathrm{s}=5)(2(\mathrm{~d}))$, the modified SMC_100 $(\mathrm{s}=$ 10) $(2(\mathrm{e}))$, the modified SMC_100 $(\mathrm{s}=20)(2(\mathrm{f}))$, and the modified SMC_100 $(\mathrm{s}=$ 50) (2(g)) algorithm simulations are shown, respectively. 

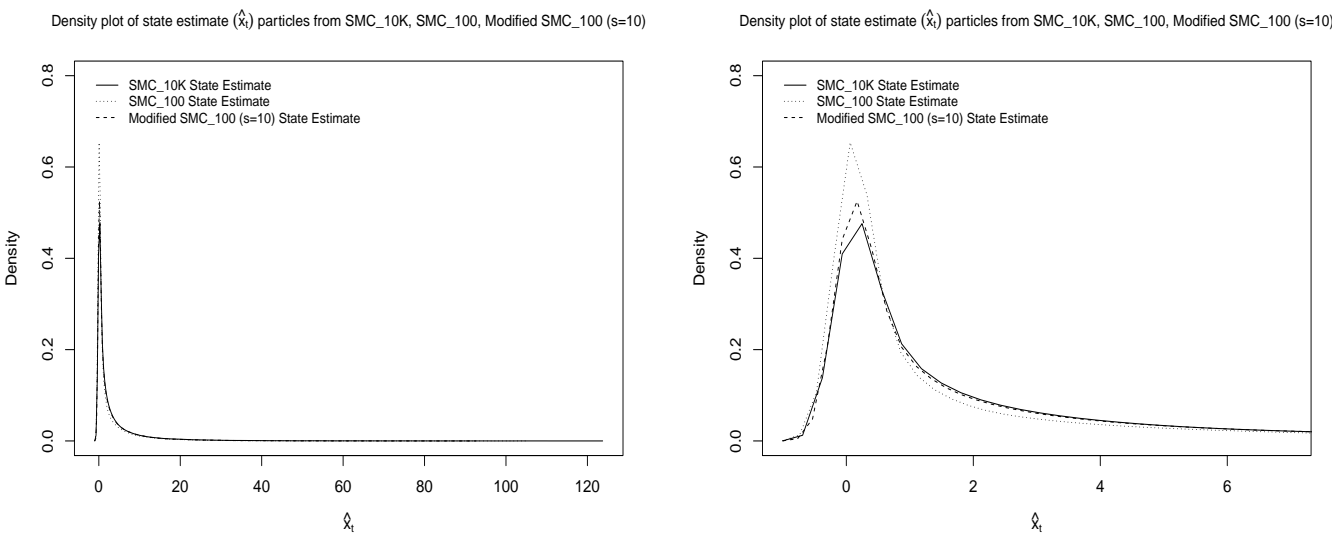

(a) limits of state estimate $\left(\hat{x}_{t}\right)$ particles (b) limits of state estimate $\left(\hat{x}_{t}\right)$ particles from $\min \hat{x}_{t}$ to $\max \hat{x}_{t}$ from $\min \hat{x}_{t}$ to $\hat{x}_{t}=7$

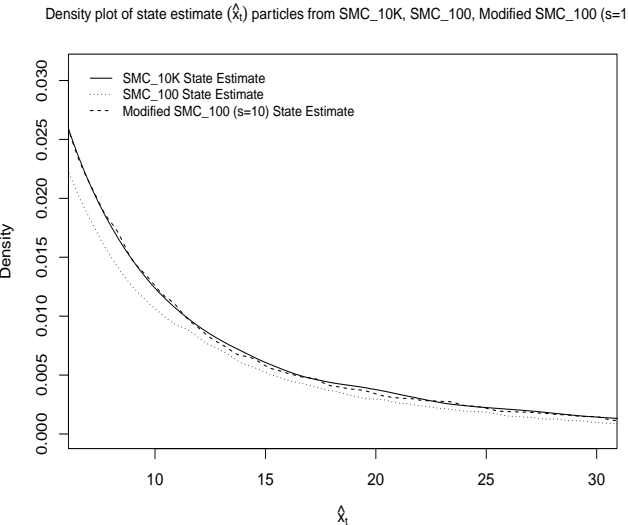

(c) limits of state estimate $\left(\hat{x}_{t}\right)$ particles from $\hat{x}_{t}=7$ to $\hat{x}_{t}=30$

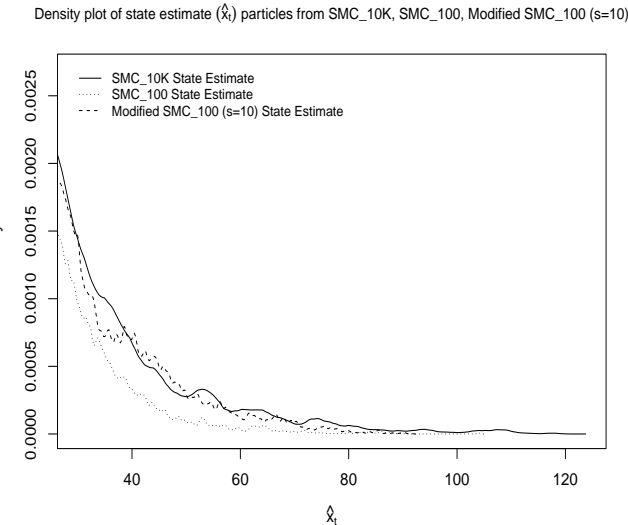

(d) limits of state estimate $\left(\hat{x}_{t}\right)$ particles from $\hat{x}_{t}=30$ to $\max \hat{x}_{t}$

Figure 3. The density plot of state estimate $\left(\hat{x}_{t}\right)$ particles obtained from the SMC_10K (solid line), the SMC_100 (dotted line), and the modified SMC_100 (s $=10)$ (dashed line) algorithm simulations with 500 repeats $(R)$, particles $(N)$, and time steps $(T)$ with various limit specifications for the state estimate $\left(\hat{x}_{t}\right)$ particles are displayed, respectively. 